\title{
ALMOST AUTOMORPHIC SOLUTIONS FOR DIFFERENTIAL EQUATIONS WITH PIECEWISE CONSTANT ARGUMENT
}

\author{
LI-LI ZHANG ${ }^{\bowtie}$ and HONG-XU LI
}

(Received 15 September 2013; accepted 25 October 2013; first published online 26 November 2013)

\begin{abstract}
Using the method of exponential dichotomies, we establish a new existence and uniqueness theorem for almost automorphic solutions of differential equations with piecewise constant argument of the form

$$
x^{\prime}(t)=A(t) x(t)+B(t) x(\lfloor t\rfloor)+f(t), \quad t \in \mathbb{R},
$$

where $\lfloor\cdot\rfloor$ denotes the greatest integer function, and $A(t), B(t): \mathbb{R} \rightarrow \mathbb{R}^{q \times q}, f(t): \mathbb{R} \rightarrow \mathbb{R}^{q}$ are all almost automorphic.

2010 Mathematics subject classification: primary 43A60; secondary 34K34.

Keywords and phrases: exponential dichotomy, almost automorphic function, almost automorphic sequence, piecewise constant argument.
\end{abstract}

\section{Introduction}

Differential equations with piecewise constant argument describe hybrid dynamical systems (a combination of continuous and discrete). These equations have the structure of continuous dynamical systems within intervals of unit length and continuous solution, and so combine properties of both differential and difference equations. They have applications in certain biomedical models and are similar in structure to those found in certain sequential continuous models of disease dynamics as treated by Busenberg and Cooke since 1982 (see [3]). Therefore there are many papers concerning differential equations with piecewise constant arguments (see $[1,7,11,16,17,19])$.

Almost periodicity and almost automorphy are attractive topics in the qualitative theory of differential equations due to their significance and applications in physics,

This work is supported by Grant No. 11372196 of NNSF of China.

(C) 2013 Australian Mathematical Publishing Association Inc. 0004-9727/2013 \$16.00 
mathematical biology, control theory and other areas. Consequently, differential equations, partial differential equations and functional differential equations with these properties have been of great interest to many authors and there is a great deal of literature on the subject (see $[5,6,9,18]$ and the references therein). It was only in 2006 [12] that the almost automorphy of solutions of differential equations with piecewise constant argument was considered. In [7, 12], by using spectral theory, the authors gave some existence and uniqueness results on the almost automorphic solutions of the two differential equations

$$
x^{\prime}(t)=A x(\lfloor t\rfloor)+f(t), \quad t \in \mathbb{R}
$$

and

$$
x^{\prime}(t)=A(t) x(\lfloor t\rfloor)+f(t), \quad t \in \mathbb{R},
$$

respectively, where $f(t)$ is an $X$-valued almost automorphic function, and $X$ is a finite dimensional Banach space.

In this paper, we consider the equation

$$
x^{\prime}(t)=A(t) x(t)+B(t) x(\lfloor t\rfloor)+f(t), \quad t \in \mathbb{R}
$$

where $A, B: \mathbb{R} \rightarrow \mathbb{R}^{q \times q}, f: \mathbb{R} \rightarrow \mathbb{R}^{q}$, and $\lfloor\cdot\rfloor$ denotes the greatest integer function. This equation was first considered by Cooke and Wiener [4] and Shah and Wiener [15]. Many papers have been devoted to various qualitative problems of quasiperiodic, almost periodic or pseudo almost periodic solutions for (1.1) (see, for example, [1, $10,16]$ and the references therein).

However, the almost automorphy of solutions of (1.1) has not yet been considered. The main purpose of this work is to establish an existence and uniqueness theorem of almost automorphic solutions of (1.1) when $A(t), B(t), f(t)$ are all almost automorphic. To facilitate this, we use the definition of 'almost automorphic sequences', and give some of their properties. Further, the theory of exponential dichotomy for difference equations plays a central role in our work.

This paper is organised as follows. In Section 2 some notation and preliminary results are presented. Our main result is first stated in Section 3.1. Then some results on the corresponding difference equations are given in Section 3.2. Finally, the main result is proved in Section 3.3.

\section{Preliminaries}

Throughout this paper, we denote by $|\cdot|$ the Euclidean norm when the argument is a vector and the corresponding operator norm when the argument is a matrix. Let $B C\left(\mathbb{R}, \mathbb{R}^{q}\right)$ be the space of bounded continuous functions $u: \mathbb{R} \rightarrow \mathbb{R}^{q}$. Equipped with the sup norm defined by $\|u\|=\sup _{t \in \mathbb{R}}|u(t)|, B C\left(\mathbb{R}, \mathbb{R}^{q}\right)$ is a Banach space. In the following, we let $\lfloor\cdot\rfloor$ denote the greatest integer function and $\langle t\rangle$ the fractional part of $t$, that is, $\langle t\rangle=t-\lfloor t\rfloor$. 


\subsection{Almost automorphic functions.}

Definition 2.1 [2]. A continuous function $f: \mathbb{R} \rightarrow \mathbb{R}^{q}$ is said to be almost automorphic if for any sequence of real numbers $\left\{s_{n}^{\prime}\right\}$, there exists a subsequence $\left\{s_{n}\right\} \subset\left\{s_{n}^{\prime}\right\}$ such that

$$
\lim _{m \rightarrow \infty} \lim _{n \rightarrow \infty} f\left(t+s_{n}-s_{m}\right)=f(t)
$$

for any $t \in \mathbb{R}$. Denote by $A A\left(\mathbb{R}^{q}\right)$ the set of all such functions.

The limit in (2.1) means that

$$
g(t)=\lim _{n \rightarrow \infty} f\left(t+s_{n}\right)
$$

is well defined for each $t \in \mathbb{R}$ and

$$
f(t)=\lim _{n \rightarrow \infty} g\left(t-s_{n}\right)
$$

for each $t \in \mathbb{R}$.

REMARK 2.2. The function $g$ is measurable and bounded, but not necessarily continuous. It is also clear from the definition above that almost periodic functions are almost automorphic. But an almost automorphic function may not be almost periodic. For example, let

$$
f(t)=\sin \frac{1}{2+\cos t+\cos \pi t}, \quad t \in \mathbb{R} .
$$

It is easy to see that $f$ is almost automorphic, but not uniformly continuous. Therefore, it is not almost periodic.

Some properties of almost automorphic functions are given in the following proposition.

Proposition 2.3 [8]. Let $f, g \in A A\left(\mathbb{R}^{q}\right)$ and l be an arbitrary real constant. Then the following statements are true:

(i) $f \pm g, l f \in A A\left(\mathbb{R}^{q}\right)$;

(ii) $\hat{f}(t):=f(-t) \in A A\left(\mathbb{R}^{q}\right)$;

(iii) $f_{l}(t):=f(t+l) \in A A\left(\mathbb{R}^{q}\right)$;

(iv) the range $R_{f}$ of $f$ is precompact, so $f$ is bounded.

It is clear that $A A\left(\mathbb{R}^{q}\right) \subset B C\left(\mathbb{R}, \mathbb{R}^{q}\right)$, and $A A\left(\mathbb{R}^{q}\right)$ is a Banach space with the norm inherited from $B C\left(\mathbb{R}, \mathbb{R}^{q}\right)$.

2.2. Almost automorphic sequences. From now on we will denote by $l^{\infty}\left(\mathbb{R}^{q}\right)$ the space of all bounded (two-sided) sequences in $\mathbb{R}^{q}$ with sup norm, that is, if $x=\{x(n)\} \in$ $l^{\infty}\left(\mathbb{R}^{q}\right)$, then

$$
\|x\|=\sup _{n \in \mathbb{Z}}|x(n)|<\infty
$$


Definition 2.4 [13]. A sequence $x \in l^{\infty}\left(\mathbb{R}^{q}\right)$ is said to be almost automorphic if for any sequence of integers $\left\{s_{k}^{\prime}\right\}$, there exists a subsequence $\left\{s_{k}\right\} \subset\left\{s_{k}^{\prime}\right\}$ such that

$$
\lim _{p \rightarrow \infty} \lim _{k \rightarrow \infty} x\left(n+s_{k}-s_{p}\right)=x(n)
$$

for any $n \in \mathbb{Z}$. Denote the set of all these sequences $x$ by $A A S\left(\mathbb{R}^{q}\right)$.

The limit in (2.2) means that

$$
y(n)=\lim _{k \rightarrow \infty} x\left(n+s_{k}\right)
$$

is well defined for each $n \in \mathbb{Z}$ and

$$
x(n)=\lim _{k \rightarrow \infty} y\left(n-s_{k}\right)
$$

for each $n \in \mathbb{Z}$.

It is clear that $A A S\left(\mathbb{R}^{q}\right)$ forms a closed subspace of $l^{\infty}\left(\mathbb{R}^{q}\right)$, and we can show that the range of an almost automorphic sequence is precompact.

By the definition of almost automorphic sequences, we can easily obtain the following proposition.

Proposition 2.5. (i) The sequence $\{x(n)\}$ is almost automorphic if and only if for any sequence of integers $\left\{s_{k}^{\prime}\right\}$, there exists a subsequence $s=\left\{s_{k}\right\} \subset\left\{s_{k}^{\prime}\right\}$ such that

$$
T_{-s} T_{s} x=x \quad \text { for each } n \in \mathbb{Z},
$$

where $T_{s} x(n)=\lim _{k \rightarrow \infty} x\left(n+s_{k}\right)$ for every $n \in \mathbb{Z}$.

(ii) $A A S\left(\mathbb{R}^{q}\right)$ is translation invariant. That is, for $x \in A A S\left(\mathbb{R}^{q}\right)$ and $k \in \mathbb{Z}$, we have $x(\cdot-k) \in A A S\left(\mathbb{R}^{q}\right)$.

Proposition 2.6. Let $\{x(n)\} \in A A S\left(\mathbb{R}^{q}\right)$, then there exists an almost automorphic function $f(t)$, such that $f(n)=x(n), n \in \mathbb{Z}$.

Proof. Let

$$
f(t)=x(n)+(t-n)(x(n+1)-x(n)), \quad t \in[n, n+1), n \in \mathbb{Z} .
$$

It is clear that $f(t)$ is continuous with $f(n)=x(n), n \in \mathbb{Z}$. We now show that $f(t)$ is almost automorphic. This requires two steps.

Step 1. Let $\left\{n_{p}^{\prime}\right\}$ be an arbitrary sequence of integers. Then there exist a subsequence $\left\{n_{p}\right\} \subset\left\{n_{p}^{\prime}\right\}$ and a sequence $\{v(n)\}$ such that

$$
\lim _{p \rightarrow \infty} x\left(n+n_{p}\right)=v(n), \quad \lim _{p \rightarrow \infty} v\left(n-n_{p}\right)=x(n), \quad \forall n \in \mathbb{Z} .
$$

Define

$$
g(t)=v(n)+(t-n)(v(n+1)-v(n)), \quad t \in[n, n+1), \quad n \in \mathbb{Z} .
$$


We now show that $\lim _{p \rightarrow \infty} f\left(t+n_{p}\right)=g(t)$. The case $\lim _{p \rightarrow \infty} g\left(t-n_{p}\right)=f(t)$ is similar, and we omit the details. From (2.3),

$$
\begin{aligned}
\lim _{p \rightarrow \infty}\left|f\left(t+n_{p}\right)-g(t)\right| \leq & \lim _{p \rightarrow \infty}\left|x\left(n+n_{p}\right)-v(n)\right| \\
& +\lim _{p \rightarrow \infty}\left|(t-n)\left(x\left(n+n_{p}+1\right)-v(n+1)\right)\right| \\
& +\lim _{p \rightarrow \infty}\left|(t-n)\left(x\left(n+n_{p}\right)-v(n)\right)\right| \\
& =0
\end{aligned}
$$

for each $t \in[n, n+1), n \in \mathbb{Z}$. Thus $\lim _{p \rightarrow \infty} f\left(t+n_{p}\right)=g(t)$.

Step 2. We now consider the general case in which $\left\{s_{p}^{\prime}\right\}$ is an arbitrary real sequence. Let $s_{p}^{\prime}=n_{p}^{\prime}+t_{p}^{\prime}$, where $n_{p}^{\prime}=\left\lfloor s_{p}^{\prime}\right\rfloor$ and $t_{p}^{\prime}=\left\langle s_{p}^{\prime}\right\rangle, p \in \mathbb{Z}$. From Step 1, we can choose a subsequence $\left\{n_{p}\right\} \subset\left\{n_{p}^{\prime}\right\}$ such that

$$
\lim _{p \rightarrow \infty} f\left(t+n_{p}\right)=g(t), \quad \lim _{p \rightarrow \infty} g\left(t-n_{p}\right)=f(t), \quad \forall t \in \mathbb{R},
$$

and $\lim _{p \rightarrow \infty} t_{p}=t_{0} \in[0,1]$. We just need to show that

$$
\lim _{p \rightarrow \infty} f\left(t+t_{p}+n_{p}\right)=\lim _{p \rightarrow \infty} f\left(t+t_{0}+n_{p}\right)=g\left(t+t_{0}\right) .
$$

It is clear that

$$
\begin{aligned}
f\left(t+t_{p}\right. & \left.+n_{p}\right)-f\left(t+t_{0}+n_{p}\right) \\
= & x\left(\left\lfloor t+t_{p}\right\rfloor+n_{p}\right)-x\left(\left\lfloor t+t_{0}\right\rfloor+n_{p}\right) \\
& +\left(\left\langle t+t_{p}\right\rangle\right)\left(x\left(\left\lfloor t+t_{p}\right\rfloor+n_{p}+1\right)-x\left(\left\lfloor t+t_{p}\right\rfloor+n_{p}\right)\right) \\
& -\left(\left\langle t+t_{0}\right\rangle\right)\left(x\left(\left\lfloor t+t_{0}\right\rfloor+n_{p}+1\right)-x\left(\left\lfloor t+t_{0}\right\rfloor+n_{p}\right)\right) .
\end{aligned}
$$

Firstly, we consider the case $\left\langle t+t_{0}\right\rangle>0$. It is easy to see that $\left\lfloor t+t_{p}\right\rfloor=\left\lfloor t+t_{0}\right\rfloor$ for sufficiently large $p$. Thus from (2.4) and the boundedness of the sequence $\{x(n)\}$, we know that

$$
\begin{aligned}
\lim _{p \rightarrow \infty} \mid & \left|f\left(t+t_{p}+n_{p}\right)-f\left(t+t_{0}+n_{p}\right)\right| \\
& =\lim _{p \rightarrow \infty}\left|\left(\left\langle t+t_{p}\right\rangle-\left\langle t+t_{0}\right\rangle\right)\left(x\left(\left\lfloor t+t_{0}\right\rfloor+n_{p}+1\right)-x\left(\left\lfloor t+t_{0}\right\rfloor+n_{p}\right)\right)\right| \\
& \leq \lim _{p \rightarrow \infty}\left|t_{p}-t_{0}\right| \cdot\left|x\left(\left\lfloor t+t_{0}\right\rfloor+n_{p}+1\right)-x\left(\left\lfloor t+t_{0}\right\rfloor+n_{p}\right)\right| \\
& =0 .
\end{aligned}
$$

We now consider the case where $\left\langle t+t_{0}\right\rangle=0$. If $t+t_{p} \rightarrow t+t_{0}+0$, then $\left\lfloor t+t_{p}\right\rfloor=$ $t+t_{0}$ for sufficiently large $p$, and $\left\langle t+t_{p}\right\rangle \rightarrow 0$, as $p \rightarrow \infty$. From (2.4),

$$
\begin{aligned}
\lim _{p \rightarrow \infty} \mid & f\left(t+t_{p}+n_{p}\right)-f\left(t+t_{0}+n_{p}\right) \mid \\
& =\lim _{p \rightarrow \infty}\left|\left\langle t+t_{p}\right\rangle\left(x\left(t+t_{0}+n_{p}+1\right)-x\left(t+t_{0}+n_{p}\right)\right)\right| \\
& =0,
\end{aligned}
$$


since $\{x(n)\}$ is bounded. If $t+t_{p} \rightarrow t+t_{0}-0$, then, for sufficiently large $p,\left\lfloor t+t_{p}\right\rfloor=$ $t+t_{0}-1$, and $\left\langle t+t_{p}\right\rangle \rightarrow 1$ as $p \rightarrow \infty$. Hence by the boundedness of $\{x(n)\}$ and (2.4), we know that

$$
\begin{aligned}
\lim _{p \rightarrow \infty} \mid & f\left(t+t_{p}+n_{p}\right)-f\left(t+t_{0}+n_{p}\right) \mid \\
= & \lim _{p \rightarrow \infty} \mid x\left(t+t_{0}+n_{p}-1\right)-x\left(t+t_{0}+n_{p}\right) \\
& \quad+\left\langle t+t_{p}\right\rangle\left(x\left(t+t_{0}+n_{p}\right)-x\left(t+t_{0}-1+n_{p}\right)\right) \mid \\
= & \lim _{p \rightarrow \infty}\left(1-\left\langle t+t_{p}\right\rangle\right)\left|x\left(t+t_{0}+n_{p}\right)-x\left(t+t_{0}-1+n_{p}\right)\right| \\
= & 0 .
\end{aligned}
$$

The proof is complete.

\section{Main result}

In the following, we assume $A(t), B(t) \in A A\left(\mathbb{R}^{q \times q}\right)$, that is, all entries of the matrices $A(t), B(t)$ are almost automorphic, and $f(t) \in A A\left(\mathbb{R}^{q}\right)$. Let $M$ be a positive constant such that $\max \{\|A\|,\|B\|,\|f\|\} \leq M$.

\subsection{Statement of our main result.}

Definition 3.1. We say that a function $x: \mathbb{R} \rightarrow \mathbb{R}^{q}$ is an almost automorphic solution of (1.1) if $x \in A A\left(\mathbb{R}^{q}\right)$, and the following two conditions are satisfied:

(i) the derivative $x^{\prime}$ of $x$ exists on $\mathbb{R}$ except possibly at the point $t=n, n \in \mathbb{Z}$, where one-sided derivatives exist;

(ii) $\quad x$ satisfies $(1.1)$ in the intervals $(n, n+1), n \in \mathbb{Z}$.

By the variation of constants formula, we know that the solution of (1.1) is

$$
x(t)=X(t) X^{-1}(n) x(n)+X(t) \int_{n}^{t} X^{-1}(u) B(u) d u x(n)+X(t) \int_{n}^{t} X^{-1}(u) f(u) d u,
$$

for $n \leq t<n+1, n \in \mathbb{Z}$, where $X(t)$ is the fundamental matrix solution of

$$
x^{\prime}(t)=A(t) x(t)
$$

with $X(0)=I$. Define

$$
G(t, s)=X(t) X^{-1}(s), \quad 0 \leq t-s \leq 1 .
$$

Then (3.1) can be rewritten as

$$
x(t)=G(t, n) x(n)+\int_{n}^{t} G(t, u) B(u) d u x(n)+\int_{n}^{t} G(t, u) f(u) d u,
$$

for $n \leq t<n+1, n \in \mathbb{Z}$. 
By the continuity of $x(t)$,

$$
\begin{aligned}
x(n+1)=G(n+ & 1, n) x(n)+\int_{n}^{n+1} G(n+1, u) B(u) d u x(n) \\
& +\int_{n}^{n+1} G(n+1, u) f(u) d u
\end{aligned}
$$

for $n \in \mathbb{Z}$. Equation (3.3) can be rewritten as

$$
x(n+1)=H(n) x(n)+h(n),
$$

where

$$
H(n)=G(n+1, n)+\int_{n}^{n+1} G(n+1, u) B(u) d u
$$

and

$$
h(n)=\int_{n}^{n+1} G(n+1, u) f(u) d u
$$

for $n \in \mathbb{Z}$. The homogeneous equation of (3.4),

$$
x(n+1)=H(n) x(n), \quad n \in \mathbb{Z},
$$

is said to admit an exponential dichotomy on $\mathbb{Z}$ if there exist positive constants $K$ and $\alpha$ and a projection $P\left(P^{2}=P\right)$ such that

$$
\begin{cases}\left|Y(n) P Y^{-1}(m)\right| \leq K e^{-\alpha(n-m)} & \text { if } n \geq m, \\ \left|Y(n)(I-P) Y^{-1}(m)\right| \leq K e^{-\alpha(m-n)} & \text { if } m \geq n,\end{cases}
$$

where $Y(n)$ is the fundamental matrix solution of (3.5) with $Y(0)=I$.

We are now in a position to state our main result.

Theorem 3.2. Assume that (3.5) admits an exponential dichotomy on $\mathbb{Z}$ with parameters $(P ; K ; \alpha)$. Then there exists a unique almost automorphic solution for (1.1).

Remark 3.3. There are many almost automorphic functions $A(t), B(t)$ such that (3.5) admits an exponential dichotomy on $\mathbb{Z}$. In fact, let

$$
A(t)=\left(\begin{array}{cc}
A_{1}(t) & 0 \\
0 & A_{2}(t)
\end{array}\right), \quad B(t)=\left(\begin{array}{cc}
B_{1}(t) & 0 \\
0 & B_{2}(t)
\end{array}\right),
$$

where

$$
\begin{aligned}
& A_{i}(t)=-3+\sin \frac{1}{2+\sin \left(\alpha_{i} t\right)+\sin \left(\alpha_{i}^{\prime} t\right)}, \\
& B_{i}(t)=\sin \frac{1}{2+\cos \left(\beta_{i} t\right)+\cos \left(\beta_{i}^{\prime} t\right)}
\end{aligned}
$$


and $\alpha_{i}, \beta_{i} \in \mathbb{Q}, \alpha_{i}^{\prime}, \beta_{i}^{\prime} \in \mathbb{R}-\mathbb{Q}, i=1,2$ (where $\mathbb{Q}$ denotes the set of rational numbers). It is easy to verify that $A(t), B(t) \in A A\left(\mathbb{R}^{2}\right)$, and

$$
S:=\sup _{n \in \mathbb{Z}}|H(n)|<1 .
$$

From [1, Theorem 15], it is clear that (3.5) admits an exponential dichotomy on $\mathbb{Z}$ with parameters $(P ; K ; \alpha)$ provided that $S<1$, where

$$
P=\mathrm{Id}, \quad K=1, \quad \alpha=\log \left(\frac{1}{S}\right) .
$$

Therefore, (3.5) admits an exponential dichotomy on $\mathbb{Z}$.

3.2. The almost automorphy of the difference equation. In the following, we assume that $H(n)$ is invertible for $n \in \mathbb{Z}$.

Lemma 3.4 [14]. Suppose that (3.5) admits an exponential dichotomy on $\mathbb{Z}$ with parameters $(P ; K ; \alpha)$, and $\{h(n)\}$ is a bounded sequence. Then (3.4) has a unique solution $\{x(n)\}$ bounded on $\mathbb{Z}$.

From [16, Proposition 8], we get that $|G(t, s)| \leq e^{M}$. We now show some properties of $G(t, s)$.

Lemma 3.5. (i) Let $\left\{s_{k}^{\prime}\right\}$ be an arbitrary real sequence. Then there exists a subsequence $\left\{s_{k}\right\} \subset\left\{s_{k}^{\prime}\right\}$, such that

$$
\begin{aligned}
& F(t, s)=\lim _{k \rightarrow \infty} G\left(t+s_{k}, s+s_{k}\right), \\
& G(t, s)=\lim _{k \rightarrow \infty} F\left(t-s_{k}, s-s_{k}\right)
\end{aligned}
$$

are well defined for each $0 \leq t-s \leq 1$.

(ii) Suppose that $\lim _{k \rightarrow \infty} t_{k}=t_{0}$. Then $\lim _{k \rightarrow \infty} G\left(t_{k}, s\right)=G\left(t_{0}, s\right)$.

Proof. (i) Since $X(t)$ is the fundamental matrix solution of (3.2), $X^{-1}(t)$ is the fundamental matrix solution of the equation

$$
x^{\prime}(t)=-x(t) A(t)
$$

Then

$$
X^{-1}(t)-X^{-1}(s)=-\int_{s}^{t} X^{-1}(u) A(u) d u, \quad t \geq s .
$$

Thus

$$
G(t, s)=X(t) X^{-1}(s)=I+\int_{s}^{t} G(t, u) A(u) d u .
$$

Let $\left\{s_{k}^{\prime}\right\}$ be an arbitrary real sequence. Since $A(t)$ is almost automorphic, there exists a subsequence $\left\{s_{k}\right\} \subset\left\{s_{k}^{\prime}\right\}$ such that

$$
\lim _{k \rightarrow \infty} A\left(t+s_{k}\right)=C(t), \quad \lim _{k \rightarrow \infty} C\left(t-s_{k}\right)=A(t), \quad \forall t \in \mathbb{R} .
$$


By the Lebesgue dominated convergence theorem, we know that

$$
\lim _{k \rightarrow \infty} \int_{s}^{t}\left|G\left(t+s_{k}, u+s_{k}\right)\right| \cdot\left|A\left(u+s_{k}\right)-C(u)\right| d u=0
$$

for each $0 \leq t-s \leq 1$. Let $Y(t)$ be the fundamental matrix solution of the equation $y^{\prime}(t)=C(t) y(t)$, and define $F(t, s)=Y(t) Y^{-1}(s), 0 \leq t-s \leq 1$. Then

$$
F(t, s)=I+\int_{s}^{t} F(t, u) C(u) d u, \quad 0 \leq t-s \leq 1 .
$$

Now, from (3.9) and (3.10),

$$
\begin{aligned}
\left|G\left(t+s_{k}, s+s_{k}\right)-F(t, s)\right|= & \left|\int_{s+s_{k}}^{t+s_{k}} G\left(t+s_{k}, u\right) A(u) d u-\int_{s}^{t} F(t, u) C(u) d u\right| \\
\leq & \int_{s}^{t}\left|G\left(t+s_{k}, u+s_{k}\right) A\left(u+s_{k}\right)-F(t, u) C(u)\right| d u \\
\leq & \int_{s}^{t}\left|G\left(t+s_{k}, u+s_{k}\right)\right| \cdot\left|A\left(u+s_{k}\right)-C(u)\right| d u \\
& +\int_{s}^{t}\left|G\left(t+s_{k}, u+s_{k}\right)-F(t, u)\right| \cdot|C(u)| d u
\end{aligned}
$$

for every $0 \leq t-s \leq 1$. Then from (3.9) and Gronwall's inequality, it follows that

$$
\lim _{k \rightarrow \infty}\left|G\left(t+s_{k}, s+s_{k}\right)-F(t, s)\right|=0 .
$$

Hence (3.6) is true. By a similar argument, we can show that (3.7) is true.

(ii) From (3.8), we know that

$$
\begin{aligned}
\left|G\left(t_{k}, s\right)-G\left(t_{0}, s\right)\right| & =\left|\int_{s}^{t_{k}} G\left(t_{k}, u\right) A(u) d u-\int_{s}^{t_{0}} G\left(t_{0}, u\right) A(u) d u\right| \\
& \leq \int_{s}^{t_{k}}\left|G\left(t_{k}, u\right)-G\left(t_{0}, u\right)\right| \cdot|A(u)| d u+M e^{M}\left|t_{k}-t_{0}\right| .
\end{aligned}
$$

Since $\lim _{k \rightarrow \infty} t_{k}=t_{0}$, by Gronwall's inequality, it follows that

$$
\lim _{k \rightarrow \infty}\left|G\left(t_{k}, s\right)-G\left(t_{0}, s\right)\right|=0
$$

The proof is complete.

Remark 3.6. From Lemma 3.5(i), it is easy to see that the sequence $\{G(n+1, n)\}$ is almost automorphic, and the function $F(t, s)$ is bounded on $0 \leq t-s \leq 1$.

Lemma 3.7. The sequences $\{h(n)\},\{H(n)\}$ are almost automorphic. 
Proof. Let $\left\{n_{k}^{\prime}\right\}$ be an arbitrary sequence of integers. From Lemma 3.5(i), there exist a subsequence $\left\{n_{k}\right\} \subset\left\{n_{k}^{\prime}\right\}$ and a function $F(t, s), 0 \leq t-s \leq 1$, such that

$$
\left\{\begin{array}{l}
\lim _{k \rightarrow \infty} f\left(t+n_{k}\right)=g(t), \quad \lim _{k \rightarrow \infty} g\left(t-n_{k}\right)=f(t), \quad \forall t \in \mathbb{R}, \\
\lim _{k \rightarrow \infty} G\left(t+n_{k}, s+n_{k}\right)=F(t, s), \quad \lim _{k \rightarrow \infty} F\left(t-n_{k}, s-n_{k}\right)=G(t, s), \\
\quad 0 \leq t-s \leq 1 .
\end{array}\right.
$$

Define

$$
h^{\prime}(n)=\int_{n}^{n+1} F(n+1, u) g(u) d u, \quad n \in \mathbb{Z} .
$$

From (3.11) and by the Lebesgue dominated convergence theorem,

$$
\begin{aligned}
\lim _{k \rightarrow \infty} \mid & h\left(n+n_{k}\right)-h^{\prime}(n) \mid \\
= & \lim _{k \rightarrow \infty}\left|\int_{n}^{n+1} G\left(n+1+n_{k}, u+n_{k}\right) f\left(u+n_{k}\right) d u-\int_{n}^{n+1} F(n+1, u) g(u) d u\right| \\
\leq & \lim _{k \rightarrow \infty} \int_{n}^{n+1}\left|G\left(n+1+n_{k}, u+n_{k}\right)-F(n+1, u)\right| \cdot\left|f\left(u+n_{k}\right)\right| d u \\
& +\lim _{k \rightarrow \infty} \int_{n}^{n+1}|F(n+1, u)| \cdot\left|f\left(u+n_{k}\right)-g(u)\right| d u \\
= & 0 .
\end{aligned}
$$

Similarly, it is easy to obtain that $\lim _{k \rightarrow \infty} h^{\prime}\left(n-n_{k}\right)=h(n)$. Thus $\{h(n)\}$ is almost automorphic. From Remark 3.6, and by a similar argument for $\{h(n)\}$, we know that $\{H(n)\}$ is almost automorphic. The proof is complete.

Theorem 3.8. Assume that (3.5) admits an exponential dichotomy on $\mathbb{Z}$ with parameters $(P, K, \alpha)$. Then there exists a unique almost automorphic solution for (3.4).

Proof. Since $\{h(n)\}$ is bounded, from Lemma 3.4, we know that (3.4) has a unique solution $\{x(n)\}$ bounded on $\mathbb{Z}$. We now show that $\{x(n)\}$ is almost automorphic. Let $\left\{s_{k}^{\prime}\right\}$ be an arbitrary sequence of integers. Since $\{H(n)\},\{h(n)\}$ are almost automorphic sequences, from Proposition 2.5, we get that there exists a subsequence $s=\left\{s_{k}\right\} \subset\left\{s_{k}^{\prime}\right\}$ such that

$$
T_{-s} T_{s} H=H, \quad T_{-s} T_{s} h=h \quad \text { pointwise on } \mathbb{Z},
$$

and $T_{-s} T_{s} x$ exists for each $n \in \mathbb{Z}$. It is easy to see that $T_{-s} T_{s} x$ is the bounded solution of

$$
y(n+1)=T_{-s} T_{s} H y(n)+T_{-s} T_{s} h(n) .
$$

By Lemma 3.4, we obtain that $T_{-s} T_{s} x=x$ for each $n \in \mathbb{Z}$. Thus from Proposition 2.5, we know $\{x(n)\}$ is almost automorphic, and the proof is complete. 


\subsection{Proof of Theorem 3.2.}

Proof. From Theorem 3.8, we know that there exists a unique almost automorphic solution $\{x(n)\}$ of (3.4). Thus by the variation of constants formula, the solution of (1.1) is

$$
x(t)=G(t, n) x(n)+\int_{n}^{t} G(t, u) B(u) d u x(n)+\int_{n}^{t} G(t, u) f(u) d u
$$

for $n \leq t<n+1, n \in \mathbb{Z}$. We now show it is almost automorphic. This requires two steps.

Step 1. We first suppose that $\left\{n_{k}^{\prime}\right\}$ is an arbitrary sequence of integers. From Lemma 3.5, we can deduce that there exist a subsequence $\left\{n_{k}\right\} \subset\left\{n_{k}^{\prime}\right\}$, a sequence $\{v(n)\}$ and a function $F(t, s), 0 \leq t-s \leq 1$, such that

$$
\left\{\begin{array}{l}
\lim _{k \rightarrow \infty} x\left(n+n_{k}\right)=v(n), \quad \lim _{k \rightarrow \infty} v\left(n-n_{k}\right)=x(n), \quad \forall n \in \mathbb{Z} ; \\
\lim _{k \rightarrow \infty} B\left(t+n_{k}\right)=C(t), \quad \lim _{k \rightarrow \infty} C\left(t-n_{k}\right)=B(t), \quad \forall t \in \mathbb{R} ; \\
\lim _{k \rightarrow \infty} f\left(t+n_{k}\right)=g(t), \quad \lim _{k \rightarrow \infty} g\left(t-n_{k}\right)=f(t), \quad \forall t \in \mathbb{R} ; \\
\lim _{k \rightarrow \infty} G\left(t+n_{k}, s+n_{k}\right)=F(t, s), \quad \lim _{k \rightarrow \infty} F\left(t-n_{k}, s-n_{k}\right)=G(t, s), \\
0 \leq t-s \leq 1 .
\end{array}\right.
$$

Define

$$
V(t)=F(t, n) v(n)+\int_{n}^{t} F(t, u) C(u) d u v(n)+\int_{n}^{t} F(t, u) g(u) d u,
$$

for $n \leq t<n+1, n \in \mathbb{Z}$.

We have

$$
\begin{aligned}
\lim _{k \rightarrow \infty} \mid & x\left(t+n_{k}\right)-V(t) \mid \\
= & \lim _{k \rightarrow \infty} \mid G\left(t+n_{k}, n+n_{k}\right) x\left(n+n_{k}\right)-F(t, n) v(n) \\
& +\int_{n}^{t} G\left(t+n_{k}, u+n_{k}\right) B\left(u+n_{k}\right) d u x\left(n+n_{k}\right)-\int_{n}^{t} F(t, u) C(u) d u v(n) \\
& +\int_{n}^{t} G\left(t+n_{k}, u+n_{k}\right) f\left(u+n_{k}\right) d u-\int_{n}^{t} F(t, u) g(u) d u \mid \\
\leq & \lim _{k \rightarrow \infty}\left|G\left(t+n_{k}, n+n_{k}\right)-F(t, n)\right| \cdot\left|x\left(n+n_{k}\right)\right|+\lim _{k \rightarrow \infty}|F(t, n)| \cdot\left|x\left(n+n_{k}\right)-v(n)\right| \\
& +\lim _{k \rightarrow \infty} \int_{n}^{t}\left|G\left(t+n_{k}, u+n_{k}\right)-F(t, u)\right| \cdot\left|B\left(u+n_{k}\right)\right| d u\left|x\left(n+n_{k}\right)\right| \\
& +\lim _{k \rightarrow \infty} \int_{n}^{t}|F(t, u)| \cdot\left|B\left(u+n_{k}\right)-C(u)\right| d u\left|x\left(n+n_{k}\right)\right|
\end{aligned}
$$




$$
\begin{aligned}
& +\lim _{k \rightarrow \infty} \int_{n}^{t}|F(t, u) C(u)| d u\left|x\left(n+n_{k}\right)-v(n)\right| \\
& +\lim _{k \rightarrow \infty} \int_{n}^{t}\left|G\left(t+n_{k}, u+n_{k}\right)-F(t, u)\right| \cdot\left|f\left(u+n_{k}\right)\right| d u \\
& +\lim _{k \rightarrow \infty} \int_{n}^{t}|F(t, u)| \cdot\left|f\left(u+n_{k}\right)-g(u)\right| d u .
\end{aligned}
$$

From (3.13), and by the Lebesgue dominated convergence theorem, we deduce that

$$
\lim _{k \rightarrow \infty}\left|x\left(t+n_{k}\right)-V(t)\right|=0, \quad \forall t \in[n, n+1), \quad n \in \mathbb{Z} .
$$

Similarly, we can deduce that $\lim _{k \rightarrow \infty} V\left(t-n_{k}\right)=x(t)$.

Step 2. We now consider the general case in which $\left\{s_{k}^{\prime}\right\}$ is an arbitrary real sequence. Let $s_{k}^{\prime}=n_{k}^{\prime}+t_{k}^{\prime}$, where $n_{k}^{\prime}=\left\lfloor s_{k}^{\prime}\right\rfloor$ and $t_{k}^{\prime}=\left\langle s_{k}^{\prime}\right\rangle, k \in \mathbb{Z}$. Then there exist a subsequence $\left\{n_{k}\right\} \subset\left\{n_{k}^{\prime}\right\}$ and a sequence $\{v(n)\}$ such that

$$
\lim _{k \rightarrow \infty} x\left(n+n_{k}\right)=v(n), \quad \lim _{k \rightarrow \infty} v\left(n-n_{k}\right)=x(n), \quad \forall n \in \mathbb{Z},
$$

and $\lim _{k \rightarrow \infty} t_{k}=t_{0} \in[0,1]$. We now show that

$$
\lim _{k \rightarrow \infty} x\left(t+t_{k}+n_{k}\right)=\lim _{k \rightarrow \infty} x\left(t+t_{0}+n_{k}\right)=v\left(t_{0}+t\right) .
$$

Firstly, we consider the case $\left\langle t+t_{0}\right\rangle>0$. It is clear that $\left\lfloor t+t_{k}\right\rfloor=\left\lfloor t+t_{0}\right\rfloor$ for sufficiently large $k$. From (3.12) and Lemma 3.5(ii), by the Lebesgue dominated convergence theorem,

$$
\begin{aligned}
\lim _{k \rightarrow \infty} \mid & x\left(t+t_{k}+n_{k}\right)-x\left(t+t_{0}+n_{k}\right) \mid \\
\leq & \lim _{k \rightarrow \infty}\left|G\left(t+t_{k}+n_{k},\left\lfloor t+t_{0}\right\rfloor+n_{k}\right)-G\left(t+t_{0}+n_{k},\left\lfloor t+t_{0}\right\rfloor+n_{k}\right)\right| \cdot\left|x\left(\left\lfloor t+t_{0}\right\rfloor+n_{k}\right)\right| \\
& +\lim _{k \rightarrow \infty} \mid \int_{\left\lfloor t+t_{0}\right\rfloor+n_{k}}^{t+t_{k}+n_{k}} G\left(t+t_{k}+n_{k}, u\right) B(u) d u x\left(\left\lfloor t+t_{0}\right\rfloor+n_{k}\right) \\
& -\int_{\left\lfloor t+t_{0}\right\rfloor+n_{k}}^{t+t_{0}+n_{k}} G\left(t+t_{0}+n_{k}, u\right) B(u) d u x\left(\left\lfloor t+t_{0}\right\rfloor+n_{k}\right) \mid \\
& +\lim _{k \rightarrow \infty}\left|\int_{\left\lfloor t+t_{0}\right\rfloor+n_{k}}^{t+t_{k}+n_{k}} G\left(t+t_{k}+n_{k}, u\right) f(u) d u-\int_{\left\lfloor t+t_{0}\right\rfloor+n_{k}}^{t+t_{0}+n_{k}} G\left(t+t_{0}+n_{k}, u\right) f(u) d u\right| \\
\leq & \lim _{k \rightarrow \infty} \int_{\left\lfloor t+t_{0}\right\rfloor+n_{k}}^{t+t_{k}+n_{k}}\left|G\left(t+t_{k}+n_{k}, u\right)-G\left(t+t_{0}+n_{k}, u\right)\right| \cdot|B(u)| d u\left|x\left(\left\lfloor t+t_{0}\right\rfloor+n_{k}\right)\right| \\
& +\lim _{k \rightarrow \infty}\left|\int_{t+t_{0}+n_{k}}^{t+t_{k}+n_{k}} G\left(t+t_{0}+n_{k}, u\right) B(u) d u\right|\left|x\left(\left\lfloor t+t_{0}\right\rfloor+n_{k}\right)\right| \\
& +\lim _{k \rightarrow \infty} \int_{\left\lfloor t+t_{0}\right\rfloor+n_{k}}^{t+t_{k}+n_{k}}\left|G\left(t+t_{k}+n_{k}, u\right)-G\left(t+t_{0}+n_{k}, u\right)\right| \cdot|f(u)| d u \\
& +\lim _{k \rightarrow \infty}\left|\int_{t+t_{0}+n_{k}}^{t+t_{k}+n_{k}} G\left(t+t_{0}+n_{k}, u\right) f(u) d u\right| \\
= & 0 .
\end{aligned}
$$


We now consider the case $\left\langle t+t_{0}\right\rangle=0$. If $t+t_{k} \rightarrow t+t_{0}+0$, then $\left\lfloor t+t_{k}\right\rfloor=\left\lfloor t+t_{0}\right\rfloor$ for sufficiently large $k$. The proof is similar to the above case. If $t+t_{k} \rightarrow t+t_{0}-0$, then, for sufficiently large $k,\left\lfloor t+t_{k}\right\rfloor=\left\lfloor t+t_{0}\right\rfloor-1$, and $\left\langle t+t_{k}\right\rangle \rightarrow 1$ as $k \rightarrow \infty$. Hence, by (3.3) and (3.12),

$$
\begin{aligned}
\lim _{k \rightarrow \infty} \mid x( & \left.+t_{k}+n_{k}\right)-x\left(t+t_{0}+n_{k}\right) \mid \\
= & \lim _{k \rightarrow \infty} \mid G\left(t+t_{k}+n_{k}, t+t_{0}-1+n_{k}\right) x\left(t+t_{0}-1+n_{k}\right)-x\left(t+t_{0}+n_{k}\right) \\
& +\int_{t+t_{0}-1+n_{k}}^{t+t_{k}+n_{k}} G\left(t+t_{k}+n_{k}, u\right) B(u) d u x\left(t+t_{0}-1+n_{k}\right) \\
& +\int_{t+t_{0}-1+n_{k}}^{t+t_{k}+n_{k}} G\left(t+t_{k}+n_{k}, u\right) f(u) d u \mid \\
\leq & \lim _{k \rightarrow \infty}\left|G\left(t+t_{k}+n_{k}, t+t_{0}-1+n_{k}\right)-G\left(t+t_{0}+n_{k}, t+t_{0}-1+n_{k}\right)\right| \\
& \times\left|x\left(t+t_{0}-1+n_{k}\right)\right| \\
& +\lim _{k \rightarrow \infty} \mid \int_{t+t_{0}-1+n_{k}}^{t+t_{k}+n_{k}} G\left(t+t_{k}+n_{k}, u\right) B(u) d u x\left(t+t_{0}-1+n_{k}\right) \\
& -\int_{t+t_{0}-1+n_{k}}^{t+t_{0}+n_{k}} G\left(t+t_{0}+n_{k}, u\right) B(u) d u x\left(t+t_{0}-1+n_{k}\right) \mid \\
& +\lim _{k \rightarrow \infty}\left|\int_{t+t_{0}-1+n_{k}}^{t+t_{k}+n_{k}} G\left(t+t_{k}+n_{k}, u\right) f(u) d u-\int_{t+t_{0}-1+n_{k}}^{t+t_{0}+n_{k}} G\left(t+t_{0}+n_{k}, u\right) f(u) d u\right| .
\end{aligned}
$$

Then by a similar argument to the above case, we know that $\lim _{k \rightarrow \infty} \mid x\left(t+t_{k}+n_{k}\right)-$ $x\left(t+t_{0}+n_{k}\right) \mid=0$. The proof is complete.

\section{References}

[1] E. Ait Dads and L. Lhachimi, 'Pseudo almost periodic solutions for equation with piecewise constant argument', J. Math. Anal. Appl. 371 (2010), 842-854.

[2] S. Bochner, 'A new approach to almost-periodicity', Proc. Nat. Acad. Sci. USA 48 (1962), 20392043.

[3] S. Busenberg and K. L. Cooke, 'Models of vertically transmitted diseases with sequentialcontinuous dynamics', in: Nonlinear Phenomena in Mathematical Sciences, (ed. V. Lakshmikantham) (Academic Press, New York, 1982), 179-187.

[4] K. L. Cooke and J. Wiener, 'Retarded differential equations with piecewise constant delays', J. Math. Anal. Appl. 99 (1984), 265-297.

[5] C. Cuevas and C. Lizama, 'Almost automorphic solutions to a class of semilinear fractional differential equations', Appl. Math. Lett. 21 (2008), 1315-1319.

[6] T. Diagana, 'Existence of globally attracting almost automorphic solutions to some nonautonomous higher-order difference equations', Appl. Math. Comput. 219 (2013), 6510-6519.

[7] W. Dimbour, 'Almost automorphic solutions for differential equations with piecewise constant argument in a Banach space', Nonlinear Anal. 74 (2011), 2351-2357.

[8] G. M. N'Guérékata, Almost Automorphy and Almost Periodic Functions in Abstract Spaces (Kluwer Academic/Plenum Publishers, New York, 2001).

[9] Y. Hino and S. Murakami, 'Almost automorphic solutions for abstract functional differential equations', J. Math. Anal. Appl. 286 (2003), 741-752. 
[10] T. Küpper and R. Yuan, 'On quasiperiodic solutions of differential equations with piecewise constant argument', J. Math. Anal. Appl. 267 (2002), 173-193.

[11] H. X. Li, 'Pseudo almost periodic sequences and some nonlinear differential equations with piecewise constant argument', Nonlinear Funct. Anal. Appl. 10 (2005), 479-493.

[12] N. V. Minh and T. T. Dat, 'On the almost automorphy of bounded solutions of differential equations with piecewise constant argument', J. Math. Anal. Appl. 326 (2007), 165-178.

[13] N. V. Minh, T. Naito and G. M. N'Guérékata, 'A spectral countability condition for almost automorphy of solutions of abstract differential equations', Proc. Amer. Math. Soc. 134 (2006), 3257-3266.

[14] K. J. Palmer, 'Exponential dichotomies, the shadowing lemma and transversal homoclinic points', Dynam. Report. 1 (1988), 265-306.

[15] S. M. Shah and J. Wiener, 'Advanced differential equations with piecewise constant argument deviations', Internat. J. Math. Soc. 6 (1983), 671-703.

[16] R. Yuan and J. L. Hong, 'The existence of almost periodic solutions for a class of differential equations with piecewise constant argument', Nonlinear Anal. 28 (1997), 1439-1450.

[17] R. Yuan, 'On Favard's theorems', J. Differential Equations 249 (2010), 1884-1916.

[18] L. L. Zhang and H. X. Li, 'Weighted pseudo-almost periodic solutions for some abstract differential equations with uniform continuity', Bull. Aust. Math. Soc. 82 (2010), 424-436.

[19] L. L. Zhang and H. X. Li, 'Weighted pseudo almost periodic solutions of second-order neutraldelay differential equations with piecewise constant argument', Comput. Math. Appl. 62 (2011), 4362-4376.

\section{LI-LI ZHANG, Department of Mathematics and Physics, \\ Shijiazhuang Tiedao University, \\ Shijiazhuang, Hebei 050043, PR China \\ e-mail: zllyou0@gmail.com}

HONG-XU LI, Department of Mathematics, Sichuan University, Chengdu, Sichuan 610064, PR China

e-mail: hoxuli@sohu.com 